\title{
artigo
}

Costa, L.F.; Medeiros, R.J.; Paungartner, L.M.; Luft, T.D.; Santos, A.P.; Paiva, T.S.; Fernandes, M.T.C.

Fatores psicossociais envolvidos na adesão ao tratamento do HIV/AIDS em adultos: revisão integrativa da literatura

\section{Insuficiencia cardíaca e as principais características definidoras do diagnóstico de enfermagem volume de líquidos excessivo}

\author{
Heart insufficiency and the main defining characteristics of nursing diagnosis excessive liquid volume \\ Insuficiencia cardíaca y las principales características que definen el diagnóstico en enfermería volumen de líquido \\ excesivo
}

\begin{abstract}
RESUMO
OBJETIVO: Analisar as principais características definidoras do diagnóstico de enfermagem Volume de Líquido Excessivo aplicado em pacientes com Insuficiência Cardíaca. MÉTODO: Foi feita pesquisa entre dezembro de 2019 e janeiro de 2020, nas plataformas eletrônicas LILACS, BDENF e SCIELO ${ }^{\circledR}$, como inclusão, foram delimitadas as publicações ocorridas entre maio de 2014 a 2019. RESULTADOS: Obteve-se uma amostra de 10 estudos, todos publicados em revistas de enfermagem, quanto ao desenho dos estudos, dois são estudos descritivos, dois são coorte, dois longitudinais, dois revisão integrativa, um transversal e um qualitativo. CONCLUSÃO: Diagnóstico com volume de líquidos excessivo e suas características são monitoradas durante a internação, sendo indicadores de qualidade dos cuidados de enfermagem da IC no hospital. Enfermeiros lidam com diversidades de sinais e sintomas no decorrer da internação que ajudam no planejamento de intervenções individualizadas e direcionadas.

DESCRITORES: Diagnóstico de enfermagem; Insuficiência cardíaca; Enfermagem.
\end{abstract}

\section{ABSTRACT}

OBJECTIVE: To analyze the main defining characteristics of the nursing diagnosis Excessive Fluid Volume applied in patients with Heart Failure. METHOD: Research was carried out between December 2019 and January 2020, on the electronic platforms LILACS, BDENF and SCIELO ${ }^{\circledR}$, as an inclusion, the publications that took place between May 2014 and 2019 were delimited. RESULTS: A sample of 10 studies was obtained, all published in nursing journals, regarding the design of the studies, two are descriptive studies, two are cohort, two are longitudinal, two are integrative reviews, one is transversal and one is qualitative. CONCLUSION: Diagnosis with excessive fluid volume and its characteristics are monitored during hospitalization, being indicators of the quality of HF nursing care at the hospital. Nurses deal with a variety of signs and symptoms during hospitalization that help in planning individualized and targeted interventions.

DESCRIPTORS: Nursing diagnosis; Cardiac insufficiency; Nursing.

\section{RESUMEN}

OBJETIVO: Analizar las principales características definitorias del diagnóstico de enfermería Volumen de líquido excesivo aplicado en pacientes con insuficiencia cardíaca. MÉTODO: La investigación se realizó entre diciembre de 2019 y enero de 2020, en las plataformas electrónicas LILACS, BDENF y ScIELO®, como inclusión, se delimitaron las publicaciones realizadas entre mayo de 2014 y 2019. RESULTADOS: Se obtuvo una muestra de 10 estudios, todos publicados en revistas de enfermería, en cuanto al diseño de los estudios, dos son estudios descriptivos, dos son de cohorte, dos son longitudinales, dos son revisiones integradoras, una es transversal y una es cualitativa. CONCLUSIÓN: El diagnóstico de exceso de volumen de líquido y sus características se monitorizan durante la hospitalización, siendo indicadores de la calidad de la atención de enfermería de la IC en el hospital. Las enfermeras se ocupan de una variedad de signos y síntomas durante la hospitalización que ayudan a planificar intervenciones individualizadas y dirigidas.

DESCRIPTORES: Diagnóstico de enfermería; Insuficiencia cardíaca; Enfermería.

RECEBIDO EM: 15/12/2020 APROVADO EM: 07/01/2021

\section{Thaís Araujo Vianna}

Acadêmica de enfermagem 7 período na Universidade Castelo Branco.

ORCID: 0000-0002-0892-5898 


\section{Kelly Cristina Freitas da Silva}

Mestre em Enfermagem pela UNIRIO na linha de Tecnologia em saúde, Docente em Enfermagem pela Universidade Castelo Branco, Enfermeira plantonista no Instituto Nacional de Cardiologia.

ORCID: 0000-0002-0882-8537

\section{Adriana Loureiro da Cunha}

Doutora em enfermagem pelo PPGENF/UERJ, docente em Enfermagem pela Universidade Castelo Branco. ORCID: 0000-0002-6971-4357

\section{Maria Regina Bernardo da Silva}

Mestre saúde da família MBA gestão em saúde, docente em Enfermagem pela Universidade Castelo Branco. ORCID: 0000-0002-3620-3091

\section{Sandra Conceição Ribeiro Chicharo}

Doutoranda paccs UFF, mestre ensino na Saúde, docente em Enfermagem pela Universidade Castelo Branco. ORCID: 0000-0002-1487-0088

\section{Alex Coelho da Silva Duarte \\ Acadêmico de enfermagem 9 período, Univeritas. ORCID: 0000-0002-1204-3943}

\section{INTRODUÇÃO}

A Insuficiência cardíaca (IC) é uma síndrome clínica resultante de qualquer injúria ao músculo cardíaco, caracterizada pela incapacidade do coração em manter um débito cardíaco dentro de valores fisiológicos para atender as necessidades tissulares. ${ }^{1}$ A Insuficiência Cardíaca Congestiva (ICC) é uma doença crônica de longo prazo que pode afetar os dois lados do coração, com isso, compromete a função de bombeamento do sangue devido ao retorno do fluxo sanguíneo, o que pode levar ao comprometimento das funções vitais, devido ao acúmulo de sangue, que resulta na falta de oxigênio para estes órgãos. ${ }^{2}$

Essa patologia carece de intervençóes terapêuticas imediatas, pois compromete a capacidade funcional de seus portadores, principalmente a IC com fração de ejeção sanguínea do ventrículo esquerdo (FEVE) reduzida que causa dispnéia, fadiga, limitam a tolerância ao exercício, além da retenção hídrica, levando à congestão pulmonar e edema periférico. Estas manifestações clínicas podem prejudicar a capacidade funcional e a qualidade de vida dos pacientes com IC. 3,4

Estudo conduzido no Brasil, demonstrou que as manifestações clínicas mais comumente apresentadas em pacientes admitidos em emergência estão relacionadas a quadros congestivos e de hipertensão. Nesse cenário, a admissão de pacientes em salas de emergência exige avaliação eficiente, rápida e segura por parte de médicos e enfermeiros. O pronto reconhecimento do quadro clínico determina e orienta as intervenções mais adequadas a esses pacientes. ${ }^{5}$

Para cuidar de pacientes com IC hospitalizados, enfermeiros estabelecem diagnósticos de enfermagem para o alcance de resultados por meio de intervençóes de enfermagem.

O Diagnóstico de Enfermagem (DE) consiste em uma das fases mais importantes do Processo de Enfermagem (PE), pois é por meio desta etapa que o profissional identifica os principais problemas vigentes no paciente, no intuito de dar início à implementação do cuidado. Porém, para que o levantamento diagnóstico seja realizado de forma satisfatória, é necessário que o enfermeiro possua um pensamento crítico fortemente embasado em evidências científicas. ${ }^{6}$

Um dos sistemas mais conhecidos e reconhecidos da etapa DE é a NANDA-I. Sua última edição data de 2018 - 2020 e é estruturada em 13 domínios, 47 classes e 244 diagnósticos. Cada diagnóstico possui título, definição, características definidoras (sinais e sintomas), fatores re- lacionados (fatores que possam causar ou contribuir para o problema) ou fatores de risco (condições que aumentam a probabilidade de aparecimento e/ou agravamento de doenças). ${ }^{7}$

Dentre os diversos DE na NANDA-I (2018), encontra-se "Volume de Líquido Excessivo" (VLE), o qual se insere no domínio 2, denominado nutrição, e na classe 5, referente à Hidratação. O VLE é definido como $\mathrm{O}$ diagnóstico de enfermagem Volume de Líquidos Excessivo é definido pela NANDA como a "retenção aumentada de líquidos isotônicos". Os fatores relacionados do diagnóstico de enfermagem Volume de Líquidos Excessivo são: mecanismo regulador comprometido, ingesta excessiva de líquidos e ingesta excessiva de sódio. O fator ingesta excessiva de líquidos, por sua vez, caracteriza-se pela ingestão maior de líquidos do que o paciente consegue eliminar. Por fim, a ingesta excessiva de sódio, quase sempre resultante de dieta inadequada, pode levar a uma sobrecarga das funções renais, ocasionando retenção de líquidos e dificultando a sua eliminação.?

As características definidoras são indicadores clínicos que se agrupam como manifestação dos diagnósticos de enfermagem. São evidências clínicas que descrevem os componentes exatos ou sinais e sintomas que representam um título diagnóstico. 


\section{artigo}

Costa, L.F.; Medeiros, R.J.; Paungartner, L.M.; Luft, T.D.; Santos, A.P.; Paiva, T.S.; Fernandes, M.T.C.

Fatores psicossociais envolvidos na adesão ao tratamento do HIV/AIDS em adultos: revisão integrativa da literatura

Resultados do grupo de pacientes com diagnóstico identificaram, como características definidoras, edema, ortopnéia, fadiga, turgência de jugular, variação de pressão arterial, balanço hídrico positivo, mudança no padrão respiratório, variação da pressão venosa central (PVC) e alteração eletrolítica, havendo concordância entre as duas peritas para os pacientes com e sem diagnóstico. ${ }^{10}$

Dados epidemiológicos demonstram que a IC é uma das principais causas de morbimortalidade dentre as doenças crônicas não transmissíveis, além de reduzir a qualidade de vida de pacientes que apresentam essa condição. ${ }^{8}$

$\mathrm{O}$ aumento da expectativa de vida e a longevidade nos países desenvolvidos têm potencialmente uma grande influência sobre a epidemiologia da ICC. Aproximadamente 23 milhões de pessoas são portadoras de ICC e 2 milhões de novos casos são diagnosticados a cada ano no mundo. Cerca de 6,5 milhões de pessoas na Europa, 5 milhões de pessoas nos Estados Unidos e 2,4 milhões de pessoas no Japão sofrem de ICC. No Brasil, estima-se que 6,4 milhões de brasileiros sofram de ICC.?

Mediante esta exposição, percebeu-se a necessidade de aprofundar o conhecimento relacionado ao uso do DE VLE. Esta investigação foi conduzida através do seguinte questionamento: Quais as características definidoras mais utilizadas para o diagnóstico de enfermagem Volume de Líquido Excessivo em pacientes com Insuficiência Cardíaca?

Torna-se necessária a realização dessa revisão integrativa para sintetizar os estudos, na tentativa de identificar as Características Definidoras mais frequentemente utilizadas nos pacientes com insuficiência cardíaca na prática clínica, bem como compará-las com as listadas pela NANDA-I. Com isso foi traçado seguinte questão norteadora: Quais as características definidoras mais utilizadas para o diagnóstico de enfermagem Volume de Líquido Excessivo em pacientes com Insuficiência Cardíaca?

\section{MÉTODO}

Trata-se de uma revisão integrativa da literatura, pois permite a síntese de estudos publicados e possibilita conclusões de uma particular área de estudo, além de apontar lacunas do conhecimento que necessitam de novos estudos. ${ }^{11}$

O presente estudo consiste em uma revisão sistemática da literatura. Dois revisores independentes (Santos e Vianna) realizaram uma seleção dos artigos inclusos na íntegra, publicados de dezembro 2019 a janeiro 2020, nos idiomas português, inglês ou espanhol, localizados na busca eletrônica meio da leitura dos títulos e resumos no período de $03 / 06 / 2018$ a $10 / 06 / 2018$. Os artigos selecionados nessa etapa foram lidos na íntegra e avaliados de acordo com os critérios de elegibilidade. Um terceiro revisor julgou se os artigos deveriam ser mantidos ou excluídos em situações em que os dois revisores discordassem. As recomendações dos Itens de Relatório Preferencial para Revisões Sistemáticas e Meta-Análises. Foi utilizado o Prisma para essa revisão.

A busca foi realizada em banco de dados eletrônicos (LILACS) Latino -Americana e do Caribe em Ciências da Saúde, (BDENF) Banco de Dados em Enfermagem e (SciELO) Scientific Electronic
Library Online e foram utilizados os seguintes descritores: "Diagnóstico de enfermagem"; "Insuficiência cardíaca"; "Enfermagem". E foi utilizada como estratégia de busca "Diagnóstico de enfermagem"; "Insuficiência cardíaca"; "Enfermagem". Como critérios de inclusão, foram delimitadas as publicações ocorridas entre maio de 2014 a 2019.

\section{RESULTADOS}

Inicialmente foram filtrados e selecionados um total de 83 artigos, disponíveis na íntegra, nos idiomas inglês e português, publicados a partir do ano de 2014. Desses, três encontravam-se duplicados e 52 foram excluídos pelo título e resumo, por não contemplarem a revisão proposta.

Portanto, foram selecionados e incluídos nesta presente revisão um total de dez artigos. Ao analisar as publicações, verifica-se que os dez artigos (100\%) foram publicados em revistas de enfermagem brasileiras.

Quanto ao desenho dos estudos, dois são estudos descritivos, dois são coorte, dois longitudinais, dois revisão integrativa, um transversal e um qualitativo. As publicações selecionadas apresentaram, em sua maioria, a temática "Diagnóstico de enfermagem em Insuficiência Cardíaca".

Este dado corrobora com as mudanças na área da educação em enfermagem no Brasil, a qual vem passando por transformações frente às exigências de seu papel na formação de recursos humanos com perfil adequado às necessidades de saúde da população e à legitimidade de seu papel na produção de conhecimentos inovadores e de utilidade para a sociedade.

QUADRO 1 - Artigos que compõem corpus da pesquisa por número de evidências, título/ano, objetivos e resultados.

\begin{tabular}{|l|l|l|l|}
\hline $\begin{array}{l}N^{\circ} \text { DE } \\
\text { EVIDÊNCIAS }\end{array}$ & TÍTULO / ANO & OBJETIVOS & RESULTADOS \\
\hline Estudo de corte & $\begin{array}{l}\text { Predição de risco e acurácia } \\
\text { diagnóstica em pacientes } \\
\text { internados com insuficiência } \\
\text { cardíaca descompensada: } \\
\text { estudo de coorte / 2019 }\end{array}$ & $\begin{array}{l}\text { Analisar a acurácia diagnóstica } \\
\text { de enfermagem em pacientes } \\
\text { com predição de risco de piora } \\
\text { clínica durante internação por } \\
\text { insuficiência cardíaca agudamen- } \\
\text { te descompensada }\end{array}$ & $\begin{array}{l}\text { Dos } 43 \text { pacientes com risco de piora a acurácia } \\
\text { diagnóstica apresentou-se na categoria } \\
\text { Moderada/Alta em 22(89\%). Débito cardíaco } \\
\text { diminuído e Volume de líquidos excessivo fo- } \\
\text { ram pontuados com 100\% na categoria Alta. }\end{array}$ \\
\hline
\end{tabular}




\begin{tabular}{|c|c|c|c|}
\hline $\begin{array}{l}\text { Estudo qualitativo } \\
\text { 3E }\end{array}$ & $\begin{array}{l}\text { Diagnósticos de enfermagem } \\
\text { para pessoas com insufici- } \\
\text { ência cardíaca: mapeamento } \\
\text { cruzado/2019 }\end{array}$ & $\begin{array}{l}\text { Mapear os enunciados diag- } \\
\text { nósticos de Enfermagem para } \\
\text { pessoas com insuficiência } \\
\text { cardíaca crônica por meio de dois } \\
\text { sistemas de classificação em } \\
\text { Enfermagem }\end{array}$ & $\begin{array}{l}\text { Possibilitou-se a o mapeamento dos enuncia- } \\
\text { dos diagnósticos para pessoas com insuficiência } \\
\text { cardíaca crônica e identificação desses nos } \\
\text { sistemas de classificação NANDA-I, Inc. e CIPE }{ }^{\circledR} \\
\text { bem como sua categorização com base nas } \\
\text { necessidades humanas básicas. }\end{array}$ \\
\hline $\begin{array}{l}\text { Estudo de corte } \\
\text { 2B }\end{array}$ & $\begin{array}{l}\text { Associação dos diagnósticos } \\
\text { de Enfermagem da NANDA } \\
\text { internacional com hospitali- } \\
\text { zação e morte em insuficiên- } \\
\text { cia cardíaca / } 2019\end{array}$ & $\begin{array}{l}\text { Identificar os diagnósticos de } \\
\text { enfermagem da NANDA-I mais } \\
\text { frequentes em uma clínica de } \\
\text { insuficiência cardíaca, verificar a } \\
\text { associação dos diagnósticos de } \\
\text { enfermagem e outras variáveis } \\
\text { independentes com o óbito. }\end{array}$ & $\begin{array}{l}\text { Foram identificados como diagnóstico de enfer- } \\
\text { magem mais frequentes ansiedade, disfunção } \\
\text { sexual, fadiga, intolerância a atividade e conhe- } \\
\text { cimento deficiente, entretanto, não apresenta- } \\
\text { ram associação com o óbito. }\end{array}$ \\
\hline $\begin{array}{l}\text { Estudo transversal } \\
4 \mathrm{E}\end{array}$ & $\begin{array}{l}\text { Diagnóstico de enfermagem } \\
\text { intolerância à atividade em } \\
\text { pacientes com insuficiência } \\
\text { cardíaca crônica / } 2019\end{array}$ & $\begin{array}{l}\text { Identificar o diagnóstico de } \\
\text { enfermagem intolerância à ativi- } \\
\text { dade da NANDA - Internacional } \\
\text { em pacientes com insuficiência } \\
\text { cardíaca crônica }\end{array}$ & $\begin{array}{l}\text { O diagnóstico de enfermagem intolerância à } \\
\text { atividade se apresenta na amostra estudada, } \\
\text { reforçando as limitações dos pacientes com } \\
\text { insuficiência cardíaca em realizar as atividades } \\
\text { de vida diária e necessidade de intervenção de } \\
\text { enfermagem para melhorar a qualidade de vida } \\
\text { dos mesmos. }\end{array}$ \\
\hline $\begin{array}{l}\text { Revisão integrativa } \\
1 \mathrm{~A}\end{array}$ & $\begin{array}{l}\text { Diagnósticos de enfermagem } \\
\text { de pacientes com insuficiên- } \\
\text { cia cardíaca com fração de } \\
\text { ejeção reduzida / } 2017\end{array}$ & $\begin{array}{l}\text { Revisar na literatura os principais } \\
\text { Diagnósticos de Enfermagem } \\
\text { (DE) em pacientes com Insufi- } \\
\text { ciência cardíaca com fração de } \\
\text { ejeção reduzida. }\end{array}$ & $\begin{array}{l}\text { Esta RI evidenciou os principais DE em pacien- } \\
\text { tes com IC e FEVE reduzida, colaborando para } \\
\text { que os cuidados clínicos sejam realizados de } \\
\text { forma específica á esta população. }\end{array}$ \\
\hline $\begin{array}{l}\text { Estudo descritivo } \\
2 \mathrm{E}\end{array}$ & $\begin{array}{l}\text { Diagnósticos e interven- } \\
\text { ções de enfermagem para } \\
\text { a pessoa com insuficiência } \\
\text { cardíaca descompensada/ } \\
2016\end{array}$ & $\begin{array}{l}\text { Identificar os diagnósticos e } \\
\text { intervenções de enfermagem à } \\
\text { pessoa com insuficiência cardía- } \\
\text { ca descompensada. }\end{array}$ & $\begin{array}{l}\text { Os termos identificados mais comuns à doença } \\
\text { foram: dispneia, edema, fadiga, débito cardíaco } \\
\text { diminuído e arritmia. Osdiagnósticos/inter- } \\
\text { venções de enfermagem contribuem para a } \\
\text { atuação do enfermeiro na tomada de decisão e } \\
\text { manejo do paciente com insufciência cardíaca } \\
\text { descompensada, possibilitando realizar uma } \\
\text { sistematização da assistência de enfermagem } \\
\text { eficaz e resolutiva. }\end{array}$ \\
\hline $\begin{array}{l}\text { Estudo longitu- } \\
\text { dinal } \\
2 \mathrm{C}\end{array}$ & $\begin{array}{l}\text { Diagnósticos de enfermagem } \\
\text { em pacientes com insuficiên- } \\
\text { cia cardíaca hospitalizados: } \\
\text { estudo longitudinal } 2016\end{array}$ & $\begin{array}{l}\text { Identificar os diagnósticos de } \\
\text { enfermagem fadiga, intolerância } \\
\text { à atividade e débito cardíaco } \\
\text { diminuído em pacientes com } \\
\text { insuficiência cardíaca hospitaliza- } \\
\text { dos e verificar a associação entre } \\
\text { as características definidoras e a } \\
\text { presença dos referidos diagnós- } \\
\text { ticos de enfermagem. }\end{array}$ & $\begin{array}{l}\text { Dos } 72 \text { pacientes, } 68,0 \% \text { eram do sexo mascu- } \\
\text { lino e apresentaram o diagnóstico de enfer- } \\
\text { magem diminuição do débito cardíaco (62,5\%) } \\
\text { na primeira semana. A fadiga apareceu apenas } \\
\text { em um paciente. Intolerância à atividade foi o } \\
\text { diagnóstico que apresentou a maior discre- } \\
\text { pância entre os especialistas. O débito cardíaco } \\
\text { diminuído foi associado às características } \\
\text { defensivas: dispnéia,edema, distensão venosa } \\
\text { jugular e fraçãa de ejeção reduzida durante as } \\
\text { três semanas de avaliação. }\end{array}$ \\
\hline $\begin{array}{l}\text { Estudo longitu- } \\
\text { dinal } \\
2 \mathrm{C}\end{array}$ & $\begin{array}{l}\text { Aplicabilidade dos resul- } \\
\text { tados de enfermagem em } \\
\text { pacientes com insuficiência } \\
\text { cardíaca e volume de líquidos } \\
\text { excessivo / } 2016\end{array}$ & $\begin{array}{l}\text { Testar a aplicabilidade clínica da } \\
\text { Nursing Outcomes Classifi cation } \\
\text { em pacientes com insuficiência } \\
\text { cardíaca descompensada e Diag- } \\
\text { nóstico de Enfermagem Volume } \\
\text { de Líquidos Excessivo. }\end{array}$ & $\begin{array}{l}\text { Foram realizadas avaliações em } 17 \text { pacientes. } \\
\text { Na avaliação clínica, mensuraram-se os resul- } \\
\text { tados de enfermagem através da avaliação de } \\
\text { seus indicadores. Seis resultados apresentaram } \\
\text { aumento nos escores, quando comparados } \\
\text { às médias da primeira e da última avaliação. A } \\
\text { utilização da Nursing Outcomes Classifi cation } \\
\text { na prática clínica demonstrou melhora dos } \\
\text { pacientes internados por insuf ciência cardíaca } \\
\text { descompensada. }\end{array}$ \\
\hline
\end{tabular}




\begin{tabular}{|c|c|c|c|}
\hline $\begin{array}{l}\text { Estudo descritivo } \\
2 \mathrm{E}\end{array}$ & $\begin{array}{l}\text { Acurácia na inferência de } \\
\text { diagnósticos de enfermagem } \\
\text { de pacientes com insuficiên- } \\
\text { cia cardíaca / } 2015\end{array}$ & $\begin{array}{l}\text { Verificar acurácia na deter- } \\
\text { minação dos diagnósticos de } \\
\text { enfermagem fadiga, intolerância } \\
\text { à atividade e débito cardíaco } \\
\text { diminuído em paciente com IC } \\
\text { hospitalizados. }\end{array}$ & $\begin{array}{l}\text { O diagnóstico de enfermagem fadiga foi o mais } \\
\text { erroneamente identificado pelos enfermeiros } \\
\text { avaliadores. A busca pelo aperfeiçoamento da } \\
\text { acurácia diagnóstica reafirma a necessidade } \\
\text { de treinamento contínuo e específico para a } \\
\text { melhora da capacidade diagnosticadora do } \\
\text { enfermeiro. }\end{array}$ \\
\hline $\begin{array}{l}\text { Revisão integrativa } \\
1 \mathrm{~A}\end{array}$ & $\begin{array}{l}\text { Diagnósticos de enfer- } \\
\text { magem de pacientes com } \\
\text { insuficiência cardíaca: revisão } \\
\text { integrativa / } 2014\end{array}$ & $\begin{array}{l}\text { Identificar o conhecimento pro- } \\
\text { duzido e publicado na literatura } \\
\text { nacional e internacional sobre } \\
\text { diagnósticos de enfermagem em } \\
\text { pacientes hospitalizados com } \\
\text { insuficiência cardíaca. }\end{array}$ & $\begin{array}{l}\text { Débito cardíaco diminuído, volume excessivo } \\
\text { de líquidos, intolerância à atividade, integridade } \\
\text { da pele prejudicada, troca de gases prejudicada, } \\
\text { conhecimento deficiente, risco de quedas e mo- } \\
\text { bilidade física prejudicada foram os diagnósticos } \\
\text { mais citados nos estudos. Os resultados vão } \\
\text { de encontro com a progressão da insuficiência } \\
\text { cardíaca, que limita a tolerância do indivíduo ao } \\
\text { exercício e causa retenção hídrica. }\end{array}$ \\
\hline \multicolumn{4}{|l|}{ Fonte: autores, 2020} \\
\hline
\end{tabular}

\section{DISCUSSÃO}

Após a análise minuciosa dos artigos foi verificado que diagnóstico do volume de líquido excessivo ainda é muito presente nos pacientes com insuficiência cardíaca e que ainda temos internações devido a esse tipo de diagnóstico.

O "volume excessivo de líquidos", na IC, tem como fator causal a ingestão excessiva de líquidos e de sódio, quase sempre resultante de uma dieta inadequada, que leva a uma sobrecarga das funções renais e retenção hídrica.

A identificação das principais características definidoras como dispneia, ortopneia, edema, refluxo hepatojugular, congestão pulmonar e pressão venosa

central elevada foram os indicadores clínicos principais para o diagnóstico volume de líquidos excessivo. Esses sinais e sintomas retratam as manifestações mais comumente apresentadas por pacientes descompensados que estão relacionadas, principalmente, a quadros congestivos. ${ }^{5}$

A característica definidora dispneia foi considerada uma das mais importantes. Trata-se de um dos sintomas mais comuns apresentados por pacientes com IC e, pode estar associado diretamente à congestão pulmonar.

A característica definidora ortopneia está associada a incapacidade de melhora da fração da função ventricular e tem sido um sintoma capaz de identificar grupos de indivíduos com pior prognóstico em longo prazo. ${ }^{5}$

\section{O "volume excessivo} de líquidos", na IC,

tem como fator

causal a ingestão

excessiva de líquidos

e de sódio, quase

sempre resultante

de uma dieta

inadequada, que leva

a uma sobrecarga

das funções renais e

retenção hídrica.

A característica definidora edema é um dado clínico relevante na avaliação, é um achado comum em pacientes com diagnóstico de IC descompensada, aponta para a necessidade de avaliação minuciosa, buscando outros sinais de congestão que possam subsidiar decisão clínica precisa. ${ }^{5}$

A identificação da presença de refluxo hepatojugular positivo é alteração que reflete a incapacidade do ventrículo direito para se adaptar ao maior volume sanguíneo que lhe é oferecido, Em pacientes com IC e dispneia, o refluxo hepatojugular é um preditor clínico utilizado, com sucesso, para identificar a IC congestiva.

A característica congestão pulmonar foi avaliada por meio do exame radiológico, salientando a importância da instrumentalização dos enfermeiros para a interpretação desse achado. Essa foi característica incluída no grupo de características principais. ${ }^{5}$

A validação da característica definidora pressão venosa central elevada também se destaca como principal. Essa medida também pode ser avaliada através da técnica de pressão venosa central estimada, obtida por meio da inspeção e mensuração da distensão jugular. ${ }^{5}$

O exame físico é um método, cujos achados podem ser de extrema utilidade na prática clínica, visto que se constitui num meio factível e de baixo custo para subsidiara avaliação de estados hemodinâmicos, especialmente em pacientes críticos.

É importante analisar o diagnóstico de enfermagem em pacientes com risco de piora clínica e devemos verificar a as- 
sociação das características definidoras ao diagnóstico de enfermagem e mapear os diagnósticos de enfermagem para pessoas com insuficiência cardíaca.

$\mathrm{O}$ volume excessivo de líquido quase sempre resulta de uma dieta inadequada, que leva a sobrecarga da função renal e retenção hídrica, os enfermeiros lidam com uma diversidade de sinais e sintomas no decorrer da internação, os resultados da NOC é preocupante e influencia o processo de enfermagem, pois o enfermeiro aponta que os resultados são mensurados de acordo com o acompanhamento clínico do paciente. ${ }^{16}$

\section{CONCLUSÃO}

A alta prevalência do diagnóstico volume de líquidos e suas características definidoras mais presentes monitoradas durante a internação podem tornar-se indicadores de qualidade dos cuidados de enfermagem no ambiente hospitalar.

Enfermeiros que cuidam de pacientes com IC lidam com uma diversidade de si- nais e sintomas no decorrer da internação que podem ajudar no planejamento de intervenções individualizadas e direcionadas.

A carência na literatura de publicações voltadas para a temática impossibilitou o

desenvolvimento pleno deste trabalho. Contudo, torna-se importante realizar revisões integrativas para classificar as publicações quanto aos níveis de evidência e, desta forma, nortear os estudantes e profissionais quanto ao assunto e o seu desenvolvimento nos últimos anos, utilizando os que possuem mais forte evidência na prática clínica.

\section{REFERENCES}

1. Bocchi Edimar Alcides, Vilas-Boas Fábio, Perrone Sergio, Caamaño Angel G, Clausell Nadine, Moreira Maria da Consolação $V$ et al. I Diretriz Latino-Americana para avaliação e conduta na insuficiência cardíaca descompensada. Arq. Bras. Cardiol. [Internet] 2005 Set [citado 2020 Dez 07]; 85(Suppl 3 ): 1-48. Disponivel em: http://www.scielo.br/scielo.php?script=sci_arttext\&pid=S0066-782X2005002200001\&lng=pt. http:// dx.doi.org/10.1590/S0066-782X2005002200001.

2. Rodriguez KL, Appelt CJ, Switzer GE, et al. They diagnosed bad heart: a qualitative exploration of patients knowledge about and experiences with heart failure. Heart Lung. 37(4):257-65, 2008.

3. Smeitzer SC, Bare B. Tratado de enfermagem médico-cirurgico. Rio de Janeiro: Guanabara Koogan 2005.

4. Bocchi EA, Marcondes FG, Bacal F, Ferraz AS, Albuquerque D, Rodrigues D, et al.; Sociedade Brasileira de Cardiologia. Atualização da diretriz brasileira de insuficiência cardíaca crônica - 2012. Arq Bras Cardiol [Internet]. 2012 [citado 2016 fev. 28]; 98(1 Supl.1):1-33. Disponivel em:

http://publicacoes.cardiol.br/consenso/2012/Diretriz\%20IC\%20 Cr\%C3\%B4nica.pdf.

5. Martins, Quenia Camille Soares et al. Volume de líquidos excessivo: validação clínica em pacientes com insuficiência cardíaca descompensada. Revista Latino-Americana de Enfermagem, v. 19, n. 3, p. 540-547, 2011.

6. Da Costa Bezerra, Angélica Laura et al. Características definidoras do diagnóstico de enfermagem débito cardíaco diminuído: revisão integrativa. Journal of Nursing UFPE/Revista de Enfermagem UFPE, v. 8, n. 5, 2014.

7. Herdman, T. Heather et al. Diagnósticos de enfermagem da NANDA-I: definições e classificação 2018-2020. Porto Alegre: Artmed, 2018.

8. Fraiha JKO, Assis AV. Insuficiência Cardíaca Aguda: Epidemiologia e Marcadores Clínicos em uma população local (ICA-EMC). Arq Catarin Med. 2012; 41(4): 63-70.

9. Freitas MTS, Puschel VAA. Heart failure: expressions of per- sonal knowledge about the disease. Rev Esc Enferm USP 2013; 47(4):919-26.

10. Rocha PCS, Maria VLR. Excesso no volume de líquidos pulmonares: diagnóstico de enfermagem. Rev Enferm UERJ. 1996;4(2):183-90.

11. Mendes KDS, Silveira RCCP, Galvão CM. Revisão integrativa: método de pesquisa para a incorporação de evidências na saúde e na enfermagem. Texto \& contexto enferm [Internet]. 2008; 17(4):758-64.

12. Pereira JMV, Cavalcanti ACD, Lopes MVO, VG Silva, Souza $\mathrm{RO}$, Gonçalves LC. Accuracy in inference of nursing diagnoses in heart failure patients. Rev Bras Enferm. 2015;68(3):690-96.

13. Ernandes, Carolina Mirapalheta et al. Predição de risco e acurácia diagnóstica em pacientes internados com insuficiência cardíaca descompensada: estudo de coorte. Revista Gaúcha de Enfermagem, v. 40, 2019.

14. Pereira, Juliana de Melo Vellozo et al. Diagnósticos de enfermagem em pacientes com insuficiência cardíaca hospitalizados: estudo Iongitudinal. Revista da Escola de Enfermagem da USP, v. 50, n. 6, p. 929-936, 2016.

15. Nascimento MNR, Silva MY da, Viana MCA, Oliveira CJ de, Martins AKL, Félix NDC. Diagnósticos de enfermagem para pessoas com insuficiência cardíaca: mapeamento cruzado. Rev enferm UFPE on line. 2019;13:e240194.

16. Araújo, Angela Amorim de; Nóbrega, Maria Miriam Lima da; Garcia, Telma Ribeiro. Diagnósticos e intervenciones de enfermería para pacientes portadores de insuficiencia cardíaca congestiva utilizando la cipe ${ }^{\circledR}$. Revista da Escola de Enfermagem da USP, v. 47, n. 2, p. 385-392, 2013.

17. De Almeida Neto, Omar Pereira. Diagnósticos de enfermagem de pacientes com insuficiência cardíaca com fração de ejeção reduzida. Rev Med Minas Gerais, v. 2017, n. 27, 1878.

18. Linhares, Joelza Celesilvia Chisté et al. Aplicabilidade dos resultados de enfermagem em pacientes com insuficiência cardíaca e volume de líquidos excessivo. Revista Gaúcha de Enfermagem, v. 37, n. 2, 2016. 